\title{
Role of Virtual Reality in Balance Training in Patients with Spinal Cord Injury: A Prospective Comparative Pre-Post Study
}

\author{
Madhusree Sengupta ${ }^{1}$, Anupam Gupta ${ }^{1}$, Meeka Khanna ${ }^{1}$, U. K. Rashmi Krishnan ${ }^{1}$, Dhritiman Chakrabarti ${ }^{2}$ \\ ${ }^{1}$ Department of Neurological Rehabilitation, National Institute of Mental Health and Neurosciences, Bangalore, India \\ ${ }^{2}$ Department of Neuro Anaesthesia, National Institute of Mental Health and Neurosciences, Bangalore, India
}

Study Design: Prospective comparative pre-post study.

Purpose: To evaluate the effects of game-based virtual reality (VR) training program for trunk postural control and balance in patients with spinal cord injury (SCI) and to assess the results according to the motor completeness (severity) of lesions using the American Spinal Injury Association Impairment Scale (AIS).

Overview of Literature: Training with VR based gaming has a role to play in improving balance in patients with SCl.

Methods: Patients with SCI (traumatic and non-traumatic) for $<6$ months were included in this hospital-based study. Participants were divided into two groups: experimental group (EG) consisting 21 patients, and control group (CG) consisting 12. Both groups underwent the conventional rehabilitation program. An additional training with semi-immersive VR therapy was conducted 5 days a week for 3 weeks in the EG with the focus on balance rehabilitation using the "Rhetoric." The outcome measures were the Berg Balance Scale (BBS), balance section of the Tinetti Performance-Oriented Mobility Assessment (POMA-B), and Functional Reach Score (FRS).

Results: Both groups consisted of young participants (mean age, 28 and 30.5 years, respectively) and predominantly men (>80\%). One-third of them had tetraplegia and two-third had paraplegia. Between-group analyses showed no statistically significant differences in the main effects between groups (p-value: BBS, 0.396; POMA-B, 0.238; FRS, 0.294), suggesting that the EG group did not show significant improvement in the trunk and posture at the end of training sessions than the CG group. Similarly, no significant difference was observed according to the severity (completeness) of SCI in the between-group analyses using the AIS (A/B vs. C/D). Conclusions: VR is an adjunctive therapy for balance rehabilitation in patients with $\mathrm{SCl}$.

Keywords: Spinal cord injuries; Virtual reality; Balance rehabilitation

\section{Introduction}

A balanced body is achieved through the coordination and integration of visual, vestibular, and proprioceptive feedback, along with the reflexive limb control [1,2]. Static postural stability, while sitting or standing, is important in performing basic activities of daily living. Dynamic balance is the ability to respond to any external stimuli or perturba-

Received Jan 17, 2019; Revised Mar 19, 2019; Accepted Apr 17, 2019

Corresponding author: Anupam Gupta

Department of Neurological Rehabilitation, National Institute of Mental Health and Neurosciences, Hosur Road, Bangalore-560029, India

Tel: +91-9480829740, Fax: +91-80-26564830,E-mail: anupam@nimhans.ac.in,drgupta159@yahoo.co.in 
tion by corrective reactions and is required for an independent wheelchair ambulation and gait performance [3].

Patients with spinal cord injury (SCI) should undergo balance rehabilitation to restore the key determinants of ambulation, such as balance, strength, muscle tone, and proprioception [4]. Additionally, normal postural synergies are lost, and sensory-motor integration of the lower limbs and trunk is impaired. This reduced spatial information contributes to balance dysfunction; therefore, patients should develop strategies using the neck, upper limb, and non-postural muscles such as the latissimus dorsi $[5,6]$.

Virtual reality (VR) is a computer-based technology that constructs a virtual environment simulating a realworld scenario and provides multimodal cues to the participants [7]. As the users interact with or manipulate the virtual objects, they receive instant visual, audio, or haptic feedback of their performances [5]. The visual stimuli can be classified depending on the extent of the user's isolation from the surroundings during an interaction. Immersive systems provide the most direct experience where a head-mounted display is often used and the visual perspective changes with the head position [7]. In semi-immersive systems, stereoscopic projections display a three-dimensional image in a fixed visual perspective. Two-dimensional presentations used in a conventional system like a desktop are known as non-immersive VR.

Recent studies suggest that VR-induced neuroplasticity promotes motor relearning, and this technology has been widely used in neurological rehabilitation [8]. In VR training, as the body's center of gravity moves beyond the base of support, proprioceptive sensations at the joints increase; therefore, participants are trained to adjust the balance perturbations [9]. Unlike the conventional training, the difficulty levels can be graded and fine-tuned by manipulating the angulation and speed of the stimulus. Hence, participants learn postural control, functional stretching of extremities, and weight shifting, which are all useful in real-life situations.

This study primarily aimed to evaluate the effects of a game-based VR training program for trunk postural control and balance in patients with SCI. The balance was also compared with a matched control group (CG) (of patients with SCI) and to assess the results with motor completeness and incompleteness in the SCI lesion using the American Spinal Injury Association Impairment Scale (AIS). AIS subgrouping was used in the analysis of associ- ated factors. We hypothesized that participants with SCI who received supplemental VR-based training along with conventional therapy will show significant improvement in static as well as dynamic balance when compared to those receiving conventional therapy alone.

\section{Materials and Methods}

This research project was approved by the Institutional Ethics Committee of National Institute of Mental Health and Neurosciences (IBR approval no., NIMH/DO/IEC [BS \& NS DIV/2017-18]).

\section{Participants}

This prospective, comparative matched pre-post study was conducted at the Department of Neurological Rehabilitation of National Institute of Mental Health and Neurosciences in Bengaluru, India, between September 2017 and May 2018. The clinical trial registration was retrospectively obtained after completing the data collection in the clinical trial registry of the United States, National Library of Medicine (protocol identifier: NCT03591497).

Participants diagnosed with SCI (both traumatic and non-traumatic etiology), aged 18-60 years with duration of illness/lesion of $<6$ months, were included in the study. Participants with inconsistent lesion severity as per the AIS scale (A, B, C, and D) were recruited [10]. Participants with a single insult, neurological level of injury (NLI) C5 or below, and ability to abduct both shoulders at $>90^{\circ}$ were recruited. Participants with visual or cognitive impairment, with severe orthopedic or neurological impairment that interferes with the short sitting, or with progressive non-traumatic SCI were excluded. Written informed consent was obtained from all participants prior to the study participation. They were blinded about the outcome measures.

\section{Calculation of the sample size}

The sample size for this study was calculated based on a pilot sample of five patients who met the inclusion criteria. Demographic details and clinical findings were recorded. Each of the participants underwent VR therapy as per the protocol along with conventional therapy. The preand post-intervention outcome measure scores from the Berg Balance Scale (BBS), balance section of the Tinetti 
Performance-Oriented Mobility Assessment (POMA-B), and Functional Reach Score (FRS) were noted. The effect sizes for pre-post differences in the outcome measures from the BBS, FRS, and POMA-B were found to be 0.81 , 1.25 , and 1.22, respectively. Assuming an acceptable alpha error of 0.5 and aiming for the $95 \%$ power of the study, the sample size for a two-tailed hypothesis was found to be 22,11 , and 11 , respectively, for the three outcomes. Hence, the largest sample size (of BBS) was selected and rounded off to 25 to compensate the attrition after recruitment. As the number of participants in both groups (21 in the EG versus 12 in the CG) is small, we believe this study can be considered as a preliminary investigation on the role of VR in balance rehabilitation in patients with SCI.

\section{Selection method}

A total of 25 patients meeting the inclusion criteria were admitted to the department for inpatient rehabilitation and included in the study. Among them, four dropped out: one due to some comorbidity that requires urgent attention (not related to SCI) and three expressed inability to stay during the entire study duration due to various reasons. Finally, 21 participants were included (in the experimental group [EG]). The participants' demographic characteristics were stratified and divided into 12 subcategories. Subsequently, 12 matched controls were recruited, each representing one subcategory. They were grouped as the CG. The EG underwent 15 sessions of VR therapy along with conventional therapy, whereas the CG underwent conventional therapy only.

\section{Matching criteria}

The controls were matched based on the following criteria: (1) neurological injury level, divided into cervical, upper thoracic, and lower thoracic; (2) etiology, traumatic and non-traumatic; (3) severity of injury, AIS 1 (motorcomplete) including AIS A and B and AIS 2 (motor-incomplete) including AIS C and D; and (4) age categorized per decade.

\section{Instrumentation}

In this study, a semi-immersive VR system known as "Rhetoric," specially designed for neurological rehabilitation by Rehametrics (Rehametrics, Valencia, Spain) was used. Microsoft Kinect (Microsoft Corp., Redmond, WA, USA) is a motion-sensing input device for the Xbox Kinect. The sensor is a horizontal bar connected to a small base mounted on a tripod, and the device features an RGB camera and depth sensor. Using these infrared camera sensors, the user's movement is detected, and an avatar is represented on the screen. The user can regulate the movement of the avatar by moving the identical body part during an interaction with the virtual environment. This movement is captured in real time, and immediate feedback is provided.

\section{Intervention}

Following the baseline assessment, routine conventional therapy consisting of individualized exercise program as per the patient's needs and goals was provided to all participants. The program was supervised by the same physiotherapist and occupational therapist. The EG received an additional course of VR. Assessors were blinded whether a participant belongs to the EG or CG.

\section{Virtual reality training}

Participants in the EG received VR training 5 days a week for 3 consecutive weeks. Each of the 15 sessions was scheduled for 30 minutes under the guidance of the same physiatrist in an isolated room without distractions. On day 0 , the concept of the program was demonstrated to the participant. All the games selected (trunk control I and II, lateral trunk displacement, bimanual coordination, antero-posterior static balance, dynamic balance I and II, static gait) focused on static and dynamic balance. Participants were expected to move their trunk and hands (as much as possible) in order to perform the activities. Games were played either while sitting on a stool or wheelchair or standing with a walker depending on the functional ability of the participant. The sessions began with a 5 -minute warm-up period and were followed by 30 minutes of gaming. Initially, the participant is allowed to rest for 1 minute for every 5 minutes of gaming. Gradually, the rest interval was decreased and gaming time increased. Every game was started at a lower level of difficulty, and the performance was scored by the software. Upon scoring $80 \%$ at each level, the participant was promoted to a higher level of difficulty. The angle or speed of the moving stimuli was changed periodically for a graded 
increase of challenges. The level of difficulty was gradually upgraded from simple to complex games based on their performance.

\section{Outcome measures}

The BBS is a 14 -item scale ranging from $0-4$ to a total score of 56 used to assess balance during functional activities $[11,12]$. The POMA-B Scale consisted nine items using a 3-point ordinal scale to measure balance and risk of fall [13].

The FRS is used to measure postural stability and dynamic balance in a sitting position $[5,14]$. The participant is instructed to sit on a stool and abduct shoulders to $90^{\circ}$. Then, the participant is asked to bend laterally as much as possible, and the difference in distance is noted for either

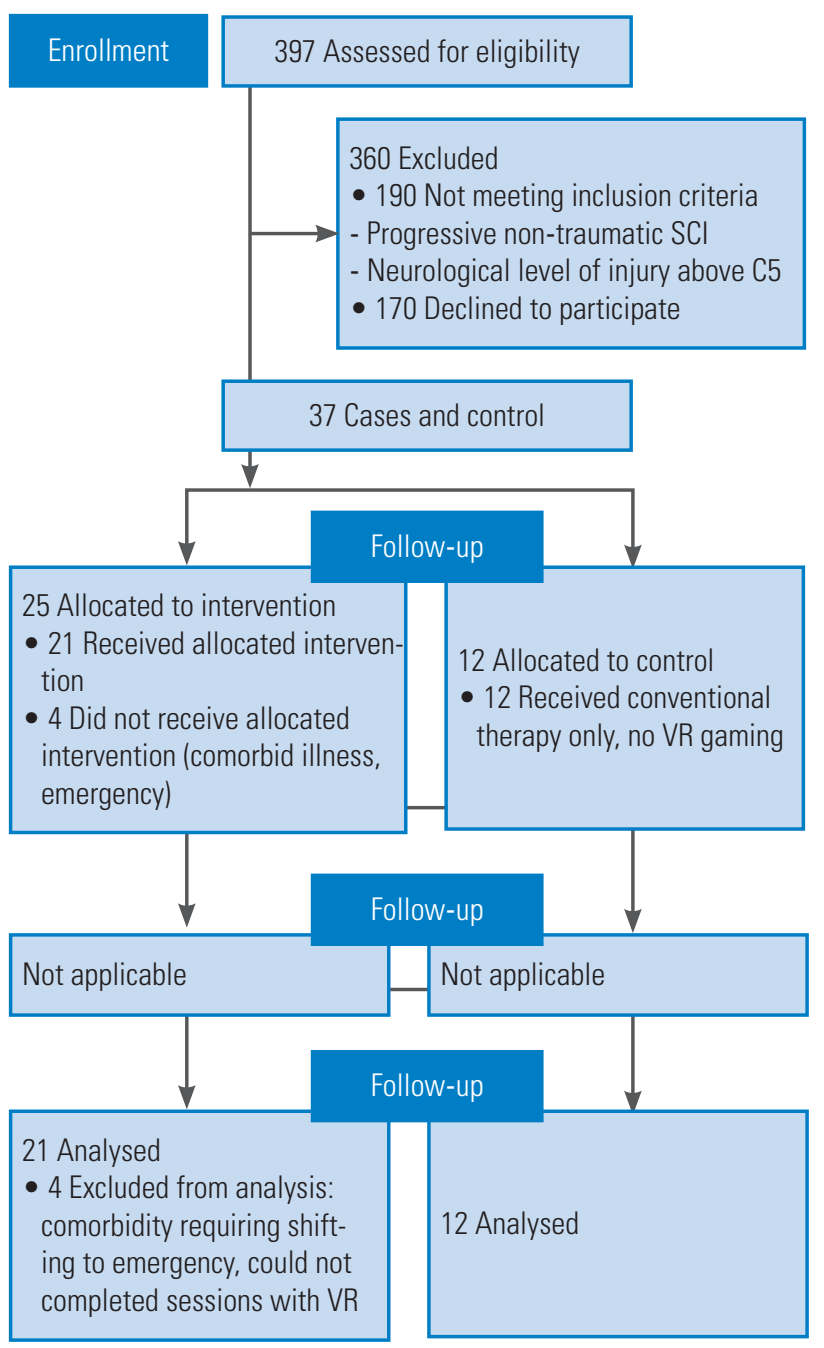

Fig. 1. Study design and flow chart. SCI, spinal cord injury; VR, virtual reality. side. Moreover, the participant is asked to bend forward with right shoulder at $90^{\circ}$ forward flexion, and the difference in distance is noted. Here the sum of the three readings is calculated and recorded (Fig. 1).

\section{Statistical analysis}

Interval scale and ordinal scale variables are presented as medians and interquartile ranges (IQR), and nominal scale data as percentages. Data were tabulated into a spreadsheet for analysis using Microsoft Excel ver. 2007 (Microsoft Corp.). Data analysis and graphical representation were conducted using the R software ver. 3.5.0 (https://www.r-project.org/). Differences in the distribution of categorical variables between groups were tested using the chi-square test or Fisher's exact test as appropriate. Due to the ordinal nature of the score variables and small sample size, non-parametric methods were used to analyze within- and between-group effects and interactions. Tests were carried out using the R package "nparLD" (https://www.r-project.org/). This method utilizes ranks of data to construct relative marginal effects for each factor level in the factorial designs and an analysis of variance test is performed to account the heteroscedastic nature of ranked data and assume an unstructured covariance structure. All $p<0.05$ were considered as the level of statistical significance.

\section{Results}

A total of 21 participants were recruited for primary objective, and after matching for pre-specified variables, 12 control participants were recruited (Fig. 1). In the EG, 17 out of 21 participants were men with a mean age of 28 years (IQR, 24-35 years). AIS A, B, C, and D were 6, 5,5 , and 5 , respectively. The NLI in the cervical, upper dorsal, and lower dorsal regions were observed in seven patients for each level. The median IQR of pre-BBS, prePOMA, and pre-FRS scores were $2(0-5), 0(0-1)$, and 33 (22-48.25), respectively.

In the CG, 10 out of 12 participants were men, with an average age of 30.5 years (IQR, 23-38 years). AIS A, B, C, and $\mathrm{D}$ were $4,3,3$, and 2, respectively. Four participants had NLI in the cervical, three in the upper dorsal, and five in the lower dorsal regions. The average pre-BBS, prePOMA, and pre-FRS scores were 1.5 (0-3.75), 0.5 (0-1), and 31 (18.25-39), respectively. No statistically significant 


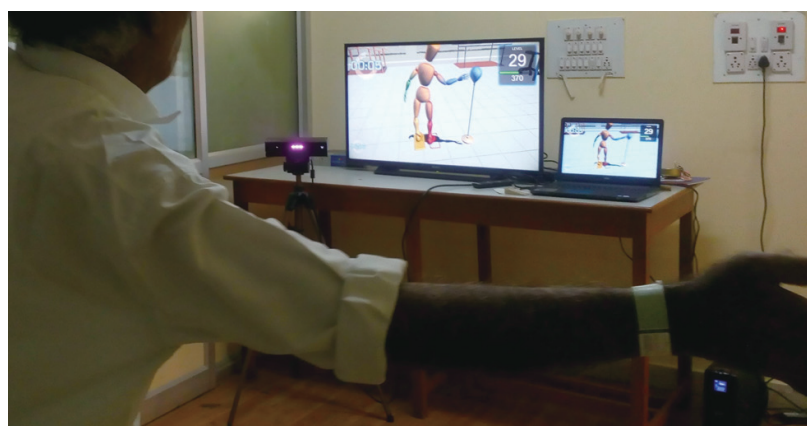

Fig. 2. Patient who underwent myelopathy training with virtual reality. difference was observed between the two groups for age $(p=0.985)$ and distribution of AIS classes $(p=0.741)$, level of injury $(p=0.4)$, and sex $(p>0.99)$. The baseline scores from the BBS, POMA-B, and FRS were also comparable between the groups ( $p>0.05$ ) (Fig. 2 ).

The results of within-group analysis are shown in Table 1. The results of between-group analysis are shown in Table 2. No statistically significant difference between the groups in the scores of pre- and post-therapy (the main effect in both groups) was observed. No major adverse events were reported by participants in either group.

Table 1. Pre-post comparison of outcome variables within the ASIA subcategories of the EG and pre-post comparison of outcome variables within the ASIA subcategories of the matched CG, respectively

\begin{tabular}{lcc} 
Variable & $\begin{array}{c}\text { At admission } \\
\text { (pre-VR therapy) }\end{array}$ & $\begin{array}{c}\text { At discharge } \\
\text { (post-VR therapy) }\end{array}$ \\
EG (cases) of ASIA 1 (ASIA A+B); EG (AIS A/B), AIS A=6, AIS B=5 & & $4(4-8)$ \\
\hline BBS & $0(0-3)$ & $2(1-3)$ \\
POMA & $0(0-1)$ & $36(30-51)$ \\
\hline FRS & $23(13.5-33)$ & 0.003 \\
CG (controls) of ASIA 1 (ASIA A+B); CG (AIS A/B), AIS A=4, AIS B=3 & & $4(3-4)$ \\
\hline BBS & $0(0-3)$ & $1(1-1)$ \\
\hline POMA & $0(0-1)$ & $34(25-46)$ \\
\hline FRS & $29(9-36)$ & 0.005 \\
\hline EG (cases) of ASIA 2 (ASIA C+D); EG (AIS C/D), AIS C=5, AIS D=5 & & 0.024 \\
\hline BBS & $3.5(1.5-6.5)$ & 0.034 \\
\hline POMA & $1(0-1.5)$ & $10(7.75-13)$ \\
\hline FRS & $48(37-50)$ & $66(52-73.5)$ \\
\hline CG (controls) of ASIA 2 (ASIA C+D); CG (AIS C/D), AIS C=3, AIS D=2 & & 0.018 \\
\hline BBS & $3(1.5-6.5)$ & 0.004 \\
\hline POMA & $1(0.5-2.5)$ & 0.005 \\
\hline FRS & $36(21.5-52)$ & $22(13-23)$ \\
\hline
\end{tabular}

Values are presented as median (interquartile range). $p<0.05$ is statistically significant.

ASIA, American Spinal Injury Association; VR, virtual reality; EG, experimental group; CG, control group; AIS, American Spinal Injury Association Impairment Scale; BBS, Berg Balance Scale; POMA, Tinetti Performance-Oriented Mobility Assessment; FRS, Functional Reach Score.

Table 2. Comparison of EG and CG after ASIA subgrouping, for pre-post differences in outcome measures

\begin{tabular}{|c|c|c|c|c|c|c|}
\hline \multirow{2}{*}{ Variable } & \multicolumn{3}{|c|}{ ASIA 1 (ASIA A/B) } & \multicolumn{3}{|c|}{ ASIA 2 (ASIA C/D) } \\
\hline & Cases & Controls & $p$-value & Cases & Controls & $p$-value \\
\hline Diff BBS & $4(2-8)$ & $4(2-4)$ & 0.285 & $19.5(13-25)$ & 18 (9.5-19) & 0.310 \\
\hline Diff POMA-B & $1(1-3)$ & $1(0-1)$ & 0.246 & $7(7-11.5)$ & $5(3-9)$ & 0.206 \\
\hline Diff FRS & $12(5.5-22)$ & $11(2-17)$ & 0.536 & $17(13.5-20.625)$ & $25(4-29.5)$ & 0.679 \\
\hline
\end{tabular}

Values are presented as median (interquartile range).

EG, experimental group; CG, control group; ASIA, American Spinal Injury Association; Diff, differences; BBS, Berg Balance Scale; POMA-B, balance section of the Tinetti Performance-Oriented Mobility Assessment; FRS, Functional Reach Score. 


\section{Discussion}

This prospective comparative pre-post study was conducted to identify the role of VR in improving the balance in patients with SCI. Participants were more responsive to learning new self-improvement strategies on receiving a visible positive feedback. Like the conventional therapy, VR training gives participants the liberty to undergo training while sitting in a wheelchair or chair, standing, and walking as a stabilized posture control is unnecessary. Only a few instances of neck and back pain were reported by postoperative participants during the initial training, which were subsequently reduced with continuous training. Two participants with cervical and one with high thoracic SCI developed orthostatic hypotension during the sessions, which was managed conservatively with medication. Participants who had a persistent postural drop in blood pressure were trained first on a tilt table and were included for the study only when asymptomatic.

The EG showed significant improvement $(p<0.001)$ in all outcome measures (BBS, POMA-B, and FRS), which have also been reported by some earlier studies [15-17].

Participants with SCI were further subgrouped according to the AIS levels for an exploratory analysis to determine if the effect of intervention on scores was influenced by the completeness of motor neurological injury. Participants with incomplete motor injury (AIS C/D) had steeper improvement in the BBS $(p=0.054)$ and POMA-B scores $(p=0.016)$ than those with AIS A/B, independent of the effects of intervention. However, the completeness of motor injury did not influence the effects of intervention on balance VR training, which has demonstrated a dynamic structural brain plasticity in the longitudinal magnetic resonance imaging using a tensor-based morphometry [18]. Full body reaching tasks to virtual targets results in greater joint excursions as compared to real-world targets. Without the actual physical end-point, one can thus move beyond the visual target $[8,14,19]$. This explains the improvement in POMA-B in the EG in our study. Villiger et al. [20] have found significant improvement in the BBS following a VR therapy in patients with incomplete SCI although the improvement was not sustainable. Considering the higher potential for ambulation in incomplete SCI, parameters such as gait speed, functionality, and quality of life have been assessed in a similar study by Wall et al. [8].

Interestingly, we POMA-B was improved in participants with motor-complete SCI. This can be explained by the following possible mechanisms. Two of the AIS A participants in the EG had recovered to AIS C during the course of treatment and VR training; therefore, motor recovery led to improved balance. This group included more AIS B participants (sensory spared) than CG. VR has been used in the proprioceptive and sensorimotor rehabilitation in the past to enhance muscle responsiveness and to stimulate recovery of motor functions $[1,7]$. In other words, incomplete SCI encompasses patients with clinically complete SCI where neurophysiological evidence of infralesional motor or somatosensory sparing can be demonstrated using a somatosensory-evoked potential or transcranial magnetic stimulation [6]. Although no tests were used to confirm the intactness of the tracts and pathways in our study, balance recovery can also be attributed to this mechanism [21].

VR training activates the mirror-neuron system [22], prefrontal cortex (PFC), parietal cortical areas, and other motor cortical networks leading to reorganization of neurons in the cerebral cortex that in turn improves balance and spatial orientation capacity and increases motion function [1]. Studies have suggested a role of VR in increasing oxygenation in the PFC of the brain while performing incremental swing balance tasks [23]. A multimodal sensory stimulus causes neural bombing on the damaged spinal cord to elicit neurophysiological and structural reorganization of the relevant pathways [22].

Although extensively used in neurorehabilitation, the validity of VR is yet to be determined. Studies on the effect of VR in SCI have been limited; most of them emphasize the positive effects of VR on mood and the need for larger sample size in validating the author observations in this study. Randomized controlled studies on SCI in a small population were conducted by Dimbwadyo-Terrer et al. [24] and Prasad et al. [22] to determine the effects of VR in the upper limb function, which have shown that VR combined with the conventional therapy yield similar results as with the conventional therapy alone. In neurological conditions such as stroke, the evidence of VR effectiveness in systematic reviews was found to be of "low quality" [25], and in multiple sclerosis [26], VR was reported to be not significantly effective as compared to the conventional therapy.

Due to the non-randomized design and small sample size in this study, the results should be cautiously interpreted. Although the CG is well matched, the matching criteria are superficial, which may lead to bias. In the ab- 
sence of follow-up, the long-term effect or sustainability of intervention could not be assessed. VR-based therapy ranges from home-based non-immersive to high-tech immersive type. A more sensitive or technically advanced software than ours/existing ones may have a different and more positive effect in balance rehabilitation. Furthermore, no guidelines were available regarding the dose, intensity, or timing of different VR-based therapy. Scales used in the study such as the POMA-B and BBS might not be sensitive to observe changes in the participants' sitting balance status. This clinical trial was registered retrospectively.

\section{Conclusions}

This study helps understand the potential benefits of VR in the balance rehabilitation of patients with SCI. It is considered as an enjoyable adjunctive therapy with a negligible side effect. VR training has not been proven to be more effective than conventional rehabilitation in this study. VR should be independently compared with the conventional therapy in this population to ensure and validate its usefulness. Further randomized, multicenter, longitudinal studies with larger sample size and for longer duration are needed to establish the role of VR in balance rehabilitation.

\section{Conflict of Interest}

No potential conflict of interest relevant to this article was reported.

\section{Acknowledgments}

The datasets generated and/or analyzed in this study are available from the corresponding author on reasonable request.

\section{Author Contributions}

MS designed the research project, supervised the intervention procedure, and wrote the manuscript. AG created the research project and evaluated and reviewed the manuscript. MK and RK provided guidance for the project. DC did the statistical analysis and wrote the results section.

\section{References}

1. Mao Y, Chen P, Li L, Huang D. Virtual reality training improves balance function. Neural Regen Res 2014;9:1628-34.

2. Lee GE, Bae H, Yoon TS, Kim JS, Yi TI, Park JS. Factors that influence quiet standing balance of patients with incomplete cervical spinal cord injuries. Ann Rehabil Med 2012;36:530-7.

3. Cho KH, Lee KJ, Song CH. Virtual-reality balance training with a video-game system improves dynamic balance in chronic stroke patients. Tohoku J Exp Med 2012;228:69-74.

4. Menon N, Gupta A, Khanna M, Taly AB. Ambulation following spinal cord injury and its correlates. Ann Indian Acad Neurol 2015;18:167-70.

5. Kizony R, Raz L, Katz N, Weingarden H, Weiss PL. Video-capture virtual reality system for patients with paraplegic spinal cord injury. J Rehabil Res Dev 2005;42:595-608.

6. Bjerkefors A, Carpenter MG, Cresswell AG, Thorstensson A. Trunk muscle activation in a person with clinically complete thoracic spinal cord injury. J Rehabil Med 2009;41:390-2.

7. Adamovich SV, Fluet GG, Tunik E, Merians AS. Sensorimotor training in virtual reality: a review. NeuroRehabilitation 2009;25:29-44.

8. Wall T, Feinn R, Chui K, Cheng MS. The effects of the Nintendo Wii Fit on gait, balance, and quality of life in individuals with incomplete spinal cord injury. J Spinal Cord Med 2015;38:777-83.

9. Lee Y, Choi W, Lee K, Song C, Lee S. Virtual reality training with three-dimensional video games improves postural balance and lower extremity strength in community-dwelling older adults. J Aging Phys Act 2017;25:621-7.

10. Roberts TT, Leonard GR, Cepela DJ. Classifications in brief: American Spinal Injury Association (ASIA) impairment scale. Clin Orthop Relat Res 2017;475:1499-504.

11. Lemay JF, Nadeau S. Standing balance assessment in ASIA D paraplegic and tetraplegic participants: concurrent validity of the Berg Balance Scale. Spinal Cord 2010;48:245-50.

12. Downs S, Marquez J, Chiarelli P. The Berg Balance Scale has high intra- and inter-rater reliability but absolute reliability varies across the scale: a systematic 
review. J Physiother 2013;59:93-9.

13. An S, Lee Y, Lee G. Validity of the performance-oriented mobility assessment in predicting fall of stroke survivors: a retrospective cohort study. Tohoku J Exp Med 2014;233:79-87.

14. Field-Fote EC, Ray SS. Seated reach distance and trunk excursion accurately reflect dynamic postural control in individuals with motor-incomplete spinal cord injury. Spinal Cord 2010;48:745-9.

15. Khurana M, Walia S, Noohu MM. Study on the effectiveness of virtual reality game-based training on balance and functional performance in individuals with paraplegia. Top Spinal Cord Inj Rehabil 2017;23:26370.

16. Tak S, Choi W, Lee S. Game-based virtual reality training improves sitting balance after spinal cord injury: a single-blinded, randomized controlled trial. Med Sci Technol 2015;56:53-9. https://doi. org/10.12659/MST.894514.

17. Betker AL, Desai A, Nett C, Kapadia N, Szturm T. Game-based exercises for dynamic short-sitting balance rehabilitation of people with chronic spinal cord and traumatic brain injuries. Phys Ther 2007;87:1389-98.

18. Villiger M, Grabher P, Hepp-Reymond MC, et al. Relationship between structural brainstem and brain plasticity and lower-limb training in spinal cord injury: a longitudinal pilot study. Front Hum Neurosci 2015;9:254.

19. Thomas JS, France CR, Leitkam ST, Applegate ME, Pidcoe PE, Walkowski S. Effects of real-world versus virtual environments on joint excursions in fullbody reaching tasks. IEEE J Transl Eng Health Med
2016;4:2100608.

20. Villiger M, Liviero J, Awai L, et al. Home-based virtual reality-augmented training improves lower limb muscle strength, balance, and functional mobility following chronic incomplete spinal cord injury. Front Neurol 2017;8:635.

21. Awad A, Levi R, Lindgren L, et al. Preserved somatosensory conduction in a patient with complete cervical spinal cord injury. J Rehabil Med 2015;47:426-31.

22. Prasad S, Aikat R, Labani S, Khanna N. Efficacy of virtual reality in upper limb rehabilitation in patients with spinal cord injury: a pilot randomized controlled trial. Asian Spine J 2018;12:927-34.

23. Basso Moro S, Bisconti S, Muthalib M, et al. A semiimmersive virtual reality incremental swing balance task activates prefrontal cortex: a functional nearinfrared spectroscopy study. Neuroimage 2014;85 Pt 1:451-60.

24. Dimbwadyo-Terrer I, Gil-Agudo A, Segura-Fragoso A, et al. Effectiveness of the virtual reality system Toyra on upper limb function in people with tetraplegia: a pilot randomized clinical trial. Biomed Res Int 2016;2016:6397828.

25. Laver KE, Lange B, George S, Deutsch JE, Saposnik G, Crotty M. Virtual reality for stroke rehabilitation. Cochrane Database Syst Rev 2017;11:CD008349.

26. Casuso-Holgado MJ, Martin-Valero R, Carazo AF, Medrano-Sanchez EM, Cortes-Vega MD, MonteroBancalero FJ. Effectiveness of virtual reality training for balance and gait rehabilitation in people with multiple sclerosis: a systematic review and metaanalysis. Clin Rehabil 2018;32:1220-34. 